\title{
Low-Frequency Electromagnetic Properties of an Alternating Stack of Thin Uniaxial Dielectric Laminae and Uniaxial Magnetic Laminae
}

\author{
Patrick S. Reese and Akhlesh Lakhtakia \\ Department of Engineering Science and Mechanics and \\ Center for the Engineering of Electronic and Acoustic Materials, The Pennsylvania State University, \\ University Park, PA, USA
}

Z. Naturforsch. 46a, 384-388 (1991); received October 20, 1990

\begin{abstract}
Analysis is carried out for an alternating arrangement of laminae which have either uniaxial permittivity or uniaxial permeability dyadics. The unit cell is constructed of two laminae such that the ones with the uniaxial permittivity dyadic alternate with the ones with the uniaxial permeability dyadic through the stack. It is assumed that both dyadics have the same optic axis. The 4-vector method is utilized to construct a characteristic matrix for the two-lamina unit cell. After assuming that each lamina is electrically thin, the properties of an equivalent medium are obtained; both the permeability and the permittivity dyadics of the equivalent medium are biaxial.
\end{abstract}

\section{Introduction}

Birefringence is the hallmark of anisotropic media. In a birefringent material, a differential phase relationship exists between the two distinctly polarized plane waves travelling in a given direction of propagation [1, 2]. This differential phase makes the use of layered anisotropic composites in devices such as filters and modulators an attractive endeavor [3].

Accurate prediction of what will happen when a plane wave is incident upon a birefringent slab does not depend solely on the optical properties of the crystal itself, but also on the vibration ellipse of the incident wave. The vibration ellipse of the transmitted wave is, in general, rotated with respect to that of the incident plane wave; further, the vibration ellipses of incident and the transmitted plane waves may not have the same eccentricities. These phenomena are jointly refered to as optical activity, because they were first observed at the optical frequencies [2, 4]. Apart from the natural optical activity exhibited by many isotropic materials of organic origin [5], optical activity is exhibited by such anisotropic materials as crystals [1], plasmas and ferrites [6], gyroelectromagnetic materials [7] and simply moving media [8,9].

Optical activity can be simulated in artificially constructed composite materials. Following an idea launched by Rumsey [10] in 1964, theoretical analyses, e.g. [4, 11], were carried out for laminated composites

Reprint requests to Prof. A. Lakhtakia, Department of Engineering Science and Mechanics, The Pennsylvania State University, 227 Hammond Building, University Park, PA 16802, USA. made from identical, electrically thin, uniaxial or biaxial laminae. In these studies, laminae were supposed to be stacked on top of each other with the consecutive optic axes describing either a left- or right-handed spiral. Such an arrangement is periodic and possesses intrinsic anisotropy as well as form chirality.

Continuing in that direction, in this paper theoretical analysis is carried out for an arrangement of laminae which have either uniaxial permittivity or uniaxial permeability dyadics. The unit cell is constructed of two laminae such that the ones with the uniaxial permittivity dyadic alternate with the ones with the uniaxial permeability dyadic throughout the stack; it is assumed that both dyadics have the same optic axis. The 4-vector method used commonly in optics is utilized to construct a characteristic matrix for the twolamina unit cell; see [4,11-14]. Finally, after assuming that the period is small in comparison to the principal wavelengths in each lamina (i.e. each lamina is electrically thin), the properties of an equivalent medium are written in terms of both types of laminae. Whereas each lamina has either an uniaxial permittivity dyadic or an uniaxial permeability dyadic, the equivalent medium is shown in general to possess a biaxial permittivity dyadic as well as a biaxial permeability dyadic.

\section{Analysis}

Consider the arrangement shown in Figure 1. The constitutive relations are given as

$$
\boldsymbol{D}=\varepsilon_{0} \boldsymbol{\varepsilon}_{n} \cdot \boldsymbol{E}, \quad \boldsymbol{B}=\mu_{0} \boldsymbol{\mu}_{n} \cdot \boldsymbol{H},
$$

0932-0784 /91/0500-0384 $\$ 01.30 / 0$. - Please order a reprint rather than making your own copy. 


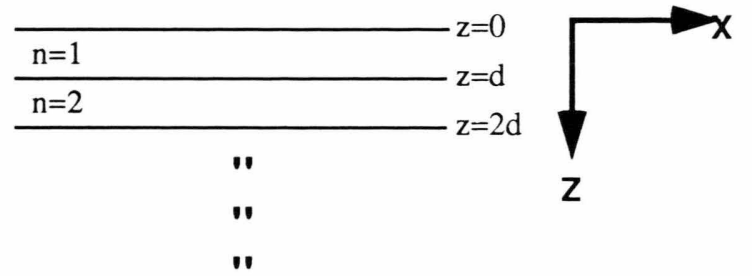

Fig. 1. Schematic of the unit cell. All optic axes, denoted by $\hat{\mathbf{c}}_{\text {subscript }}$, lie in the $x-y$ plane.

where $\varepsilon_{n}$ and $\boldsymbol{\mu}_{n}$ are the usual relative permittivity and relative permeability dyadics in the $n$th lamina. Each lamina is of thickness $d$ with $\boldsymbol{\varepsilon}_{n}=\boldsymbol{\varepsilon}_{n+2}$ and $\boldsymbol{\mu}_{n}=\boldsymbol{\mu}_{n+2}$, where

$$
\begin{aligned}
& \boldsymbol{\varepsilon}_{1}=\varepsilon_{1 \perp} \mathbf{I}+\left(\varepsilon_{1 \|}-\varepsilon_{1 \perp}\right) \hat{\mathbf{c}} \hat{\mathbf{c}}, \quad \boldsymbol{\mu}_{1}=\mu_{1} \mathbf{I}, \\
& \boldsymbol{\varepsilon}_{2}=\varepsilon_{2} \mathbf{I}, \quad \boldsymbol{\mu}_{2}=\mu_{2 \perp} \mathbf{I}+\left(\mu_{2 \|}-\mu_{2 \perp}\right) \hat{\mathbf{c}} \hat{\mathbf{c}}
\end{aligned}
$$

with $\mathbf{I}$ being the identity dyadic, while

$$
\hat{\mathbf{c}}=\hat{\boldsymbol{x}} \cos \alpha+\hat{\boldsymbol{y}} \sin \alpha,
$$

showing that the (common) preferred axis of the constitutive dyadics $\varepsilon_{1}$ and $\boldsymbol{\mu}_{2}$ lies in the $x-y$ plane. It is also assumed that $\varepsilon_{1 \perp}, \varepsilon_{1 \|}, \varepsilon_{2}, \mu_{2 \perp}, \mu_{2 \|}$, and $\mu_{1}$ are all real positive.

Pertinent to the present problem, and using an $\exp (-i \omega t)$ time-dependence, both $\boldsymbol{E}$ and $\boldsymbol{H}$ are decomposed as $[4,11]$

$$
\begin{aligned}
& \boldsymbol{E}=\boldsymbol{e}(z) e^{i \varkappa x}=\left[e_{x}(z) \hat{\boldsymbol{x}}+e_{y}(z) \hat{\boldsymbol{y}}+e_{z}(z) \hat{z}\right] e^{i \varkappa x}, \\
& \boldsymbol{H}=\boldsymbol{h}(z) e^{i \varkappa x}=\left[h_{x}(z) \hat{\boldsymbol{x}}+h_{y}(z) \hat{\boldsymbol{y}}+h_{z}(z) \hat{z}\right] e^{i \varkappa x},
\end{aligned}
$$

in which $x$ is the horizontal wavenumber mandated by Snel's laws, while the unit Cartesian vectors are denoted by $\hat{\boldsymbol{x}}, \hat{\boldsymbol{y}}$, and $\hat{\boldsymbol{z}}$. Let a 4 -vector $\boldsymbol{f}$ be defined such that

$$
f(z)=\operatorname{column}\left[e_{x}(z), e_{y}(z), h_{x}(z), h_{y}(z)\right] ;
$$

thus, the Maxwell curl equations can be compactly written as

$$
(\mathrm{d} / \mathrm{d} z) \boldsymbol{f}=\mathrm{i} \mathbf{P}_{n} \cdot \boldsymbol{f}(z)
$$

in the $n$th lamina, where $\mathbf{P}_{n}=\mathbf{P}_{n+2}, n=1,2,3, \ldots$, are $4 \times 4$ matrices given as

$$
\boldsymbol{P}_{1}=\left(\begin{array}{cccc}
0 & 0 & 0 & -\chi^{2} / \omega \varepsilon_{0} \varepsilon_{1 \perp}+\omega \mu_{0} \mu_{1} \\
0 & 0 & -\omega \mu_{0} \mu_{1} & 0 \\
\omega \varepsilon_{0}\left(\varepsilon_{1 \perp}-\varepsilon_{1 \|}\right)(\cos \alpha \sin \alpha) & \chi^{2} / \omega \mu_{0} \mu_{1}+\omega \varepsilon_{0}\left[\left(\varepsilon_{1 \perp}-\varepsilon_{1 \|}\right) \sin ^{2} \alpha-\varepsilon_{1 \perp}\right] & 0 & 0 \\
\left.\omega \varepsilon_{0}\left[\varepsilon_{1 \|}-\varepsilon_{1 \perp}\right) \cos ^{2} \alpha+\varepsilon_{1 \perp}\right] & \omega \varepsilon_{0}\left(\varepsilon_{1 \|}-\varepsilon_{1 \perp}\right)(\cos \alpha \sin \alpha) & 0 & 0
\end{array}\right)
$$

and

$$
\boldsymbol{P}_{2}=\left(\begin{array}{cccc}
0 & 0 & \omega \mu_{0}\left(\mu_{2 \|}-\mu_{2 \perp}\right)(\cos \alpha \sin \alpha) & -\varkappa^{2} / \omega \varepsilon_{0} \varepsilon_{2}+\omega \mu_{0}\left[\left(\mu_{2 \|}-\mu_{2 \perp}\right) \sin ^{2} \alpha+\mu_{2 \perp}\right] \\
0 & 0 & \omega \mu_{0}\left[\left(\mu_{2 \perp}-\mu_{2 \|}\right) \cos ^{2} \alpha-\mu_{2 \perp}\right] & \omega \mu_{0}\left(\mu_{2 \perp}-\mu_{2 \|}\right)(\cos \alpha \sin \alpha) \\
0 & \chi^{2} / \omega \mu_{0} \mu_{2 \perp}-\omega \varepsilon_{0} \varepsilon_{2} & 0 & 0 \\
\omega \varepsilon_{0} \varepsilon_{2} & 0 & 0 & 0
\end{array}\right) .
$$


$t_{1,23}=-t_{1,24}=-\left\{\omega \varepsilon_{0} \varepsilon_{1 \perp}^{1 / 2}\left[\omega^{2} \varepsilon_{0} \varepsilon_{1 \perp} \varepsilon_{1} \| \mu_{0} \mu_{1} \quad(13 \mathrm{~d})\right.\right.$

$\left.\left.-\left(\varepsilon_{1 \perp} \sin ^{2} \alpha+\varepsilon_{1||} \cos ^{2} \alpha\right) \chi^{2}\right]^{1 / 2}\right\} /\left(\omega^{2} \varepsilon_{1 \perp} \varepsilon_{0} \mu_{1} \mu_{0}-\varkappa^{2}\right)$,

$t_{1,31}=-t_{1,32}$

$=-\left[\left(\omega^{2} \varepsilon_{1 \perp} \varepsilon_{0} \mu_{1} \mu_{0}-\chi^{2}\right)^{1 / 2} \cot \alpha\right] /\left(\omega \mu_{1} \mu_{0}\right)$,

$t_{1,21}=t_{1,42}=-\cot \alpha$,

$t_{1,33}=-t_{1,34}=\left\{2 \omega \varepsilon_{0} \varepsilon_{1 \perp} \sin ^{2} \alpha\left[\omega^{2} \varepsilon_{0} \varepsilon_{1 \|} \mu_{0} \mu_{1}-\chi^{2}\right.\right.$

$$
\left.\left.+\varkappa^{2} \cos ^{2} \alpha\left(1-\varepsilon_{1 \|} / \varepsilon_{1 \perp}\right)\right]^{1 / 2}\right\} /
$$$$
/\left[\left(\omega^{2} \varepsilon_{1 \perp} \varepsilon_{0} \mu_{1} \mu_{0}-\varkappa^{2}\right) \sin 2 \alpha\right] \text {, }
$$

$t_{1,43}=t_{1,44}=2 \omega^{2} \varepsilon_{1 \perp} \varepsilon_{0} \mu_{1} \mu_{0} \sin ^{2} \alpha /$

$$
/\left[\left(\omega^{2} \varepsilon_{1 \perp} \varepsilon_{0} \mu_{1} \mu_{0}-\varkappa^{2}\right) \sin 2 \alpha\right]
$$

$t_{2,31}=t_{2,32}=t_{2,33}=t_{2,34}=1$,

$t_{2,42}=-t_{2,41}=-\left(\omega \mu_{2 \perp} \mu_{0}\right) /\left(\omega^{2} \mu_{2 \perp} \mu_{0} \varepsilon_{2} \varepsilon_{0}-x^{2}\right)^{1 / 2}$,

$t_{2,43}=-t_{2,44}=\left\{\omega \mu_{0} \mu_{2}^{1 / 2}\left[\omega^{2} \mu_{0} \mu_{2 \perp} \mu_{2 \|} \varepsilon_{0} \varepsilon_{2}\right.\right.$

$\left.\left.-\left(\mu_{2 \perp} \sin ^{2} \alpha+\mu_{2 \|} \cos ^{2} \alpha\right) \chi^{2}\right]^{1 / 2}\right\} /\left(\omega^{2} \mu_{2 \perp} \mu_{0} \varepsilon_{2} \varepsilon_{0}-\varkappa^{2}\right)$,

$t_{2,11}=-t_{2,12}=\left[\left(\omega^{2} \mu_{2} \perp \mu_{0} \varepsilon_{2} \varepsilon_{0}-\varkappa^{2}\right)^{1 / 2} \cot \alpha\right] /\left(\omega \varepsilon_{2} \varepsilon_{0}\right)$,

$t_{2,21}=t_{2,22}=-\cot \alpha$,

$t_{2,13}=-t_{2,14}=-\left\{2 \omega \mu_{0} \mu_{2 \perp} \sin ^{2} \alpha[\omega)^{2} \mu_{0} \mu_{2 \|} \varepsilon_{0} \varepsilon_{2}\right.$

$$
\left.\left.-x^{2}+x^{2} \cos ^{2} \alpha\left(1-\mu_{2 \|} / \mu_{2 \perp}\right)\right]^{1 / 2}\right\} /
$$

$/\left[\left(\omega^{2} \mu_{2} \perp \mu_{0} \varepsilon_{2} \varepsilon_{0}-\chi^{2}\right) \sin 2 \alpha\right]$,

$t_{2,23}=t_{2,24}=-2 \omega^{2} \mu_{2 \perp} \mu_{0} \varepsilon_{2} \varepsilon_{0} \sin ^{2} \alpha$

$$
/\left[\left(\omega^{2} \mu_{2} \perp \mu_{0} \varepsilon_{2} \varepsilon_{0}-\chi^{2}\right) \sin 2 \alpha\right] .
$$

\section{The Long-Wavelength Approximation}

At this point, a long-wavelength approximation may be applicable. In this approximation, the

$\mathbf{P}_{\mathrm{EQ}}=\left(\begin{array}{cc}0 & 0 \\ 0 & 0 \\ -\omega \varepsilon_{0} \varepsilon_{\mathrm{b}} \sin \left(\alpha_{1}+\alpha_{2}\right) & -\omega \varepsilon_{0} \varepsilon_{\mathrm{a}} D_{\mathrm{EQ}}-2 \omega \varepsilon_{0} \varepsilon_{\mathrm{b}} \sin \alpha_{1} \sin \alpha_{2} \\ \omega \varepsilon_{0} \varepsilon_{\mathrm{a}}+2 \omega \varepsilon_{0} \varepsilon_{\mathrm{b}} \cos \alpha_{1} \cos \alpha_{2} & \omega \varepsilon_{0} \varepsilon_{\mathrm{b}} \sin \left(\alpha_{1}+\alpha_{2}\right)\end{array}\right.$

$\left(g_{n 1}, g_{n 2}\right)$ of $\mathbf{P}_{n}$ are small enough to assume $\left|2 g_{n 1} d\right| \ll 1$ and $\left|2 g_{n 2} d\right| \ll 1$ for $n=1,2$. Therefore, on the right hand side of (10) the approximation

$$
\left.\exp \left(i \mathbf{P}_{2} d\right) \cdot \exp \left(i \mathbf{P}_{1} d\right) \approx \exp \left[i \mathbf{P}_{2}+\mathbf{P}_{1}\right) d\right]
$$

may be used. As with any approximate method, a certain amount of error is thereby introduced. This error, correct to order $d^{3}$, is given by

$$
\begin{aligned}
\boldsymbol{\Delta}_{\mathrm{e}}= & {\left[\exp \left(i \mathbf{P}_{1} d\right)\left[\exp \left(i \mathbf{P}_{2} d\right)\right]-\exp \left[i\left(\mathbf{P}_{1}+\mathbf{P}_{2}\right) d\right]\right.} \\
= & (1 / 2)\left[\mathbf{P}_{1} \mathbf{P}_{2}-\mathbf{P}_{2} \mathbf{P}_{1}\right] d^{2}+(1 / 6)\left[\left(\mathbf{P}_{1} \mathbf{P}_{2}-\mathbf{P}_{2} \mathbf{P}_{1}\right) \mathbf{P}_{2}\right. \\
& +\mathbf{P}_{1}\left(\mathbf{P}_{1} \mathbf{P}_{2}-\mathbf{P}_{2} \mathbf{P}_{1}\right)+\mathbf{P}_{1}\left(\mathbf{P}_{1}+\mathbf{P}_{2}\right) \mathbf{P}_{2} \\
& \left.-\mathbf{P}_{2}\left(\mathbf{P}_{1}+\mathbf{P}_{2}\right) \mathbf{P}_{1}\right] d^{3} .
\end{aligned}
$$

Since $\boldsymbol{\Delta}_{\mathrm{c}}=0$ correct to order $d$, the applicability of the approximation (14) is justifiable provided the conditions first stated are met. Parenthetically, it should be noted that if $\mathbf{P}_{1}=\mathbf{P}_{2}$ then the right hand side of (15) is identically zero, and a similar situation also results if $\mathbf{P}_{1}$ and $\mathbf{P}_{2}$ commute. For the present purposes, however, $\mathbf{P}_{1}$ and $\mathbf{P}_{2}$ do not commute in general.

Now the properties of the stack of the thin uniaxial laminae can be related to the properties of an equivalent medium within the limitations of the approximation. The form of the matrix $\left(\mathbf{P}_{1}+\mathbf{P}_{2}\right)$ suggests that the equivalent medium is gyroelectromagnetic with biaxial permittivity and permeability dyadics [11]. Thus

$$
\boldsymbol{D}=\varepsilon_{0} \boldsymbol{\varepsilon}_{\mathrm{EQ}} \cdot \boldsymbol{E}, \quad \boldsymbol{B}=\mu_{0} \boldsymbol{\mu}_{\mathrm{EQ}} \cdot \boldsymbol{H}
$$

are the constitutive relations for the equivalent medium, where

$$
\begin{aligned}
& \boldsymbol{\varepsilon}_{\mathrm{EQ}}=\varepsilon_{\mathrm{a}} \mathbf{I}+\varepsilon_{\mathrm{b}}\left(\hat{\mathbf{c}}_{\varepsilon 1} \hat{\mathbf{c}}_{\varepsilon 2}+\hat{\mathbf{c}}_{\varepsilon 2} \hat{\mathbf{c}}_{\varepsilon 1}\right), \\
& \boldsymbol{\mu}_{\mathrm{EQ}}=\mu_{\mathrm{a}} \mathbf{I}+\boldsymbol{\mu}_{\mathrm{b}}\left(\hat{\mathbf{c}}_{\mu 1} \hat{\mathbf{c}}_{\mu 2}+\hat{\mathbf{c}}_{\mu 2} \hat{\mathbf{c}}_{\mu 1}\right), \\
& \hat{\mathbf{c}}_{\varepsilon 1}=\hat{\boldsymbol{x}} \cos \alpha_{1}+\hat{\boldsymbol{y}} \sin \alpha_{1}, \\
& \hat{\mathbf{c}}_{\varepsilon 2}=\hat{\boldsymbol{x}} \cos \alpha_{2}+\hat{\boldsymbol{y}} \sin \alpha_{2}, \\
& \hat{\mathbf{c}}_{\mu 1}=\hat{\boldsymbol{x}} \cos \beta_{1}+\hat{\boldsymbol{y}} \sin \beta_{1}, \\
& \hat{\mathbf{c}}_{\mu 2}=\hat{\boldsymbol{x}} \cos \beta_{2}+\hat{\boldsymbol{y}} \sin \beta_{2} .
\end{aligned}
$$

In the equivalent medium, the counterpart of $(\mathrm{d} / \mathrm{d} z) f$ $=i \mathbf{P}_{n} \cdot \boldsymbol{f}(z)$ is $(\mathrm{d} / \mathrm{d} z) \boldsymbol{f}=i \mathbf{P}_{\mathrm{EQ}} \cdot \boldsymbol{f}(z)$, where [11]

$$
\begin{array}{cc}
\omega \mu_{0} \mu_{\mathrm{b}} \sin \left(\beta_{1}+\beta_{2}\right) & \omega \mu_{0} \mu_{\mathrm{a}} D_{\mathrm{EQ}}+2 \omega \mu_{0} \mu_{\mathrm{b}} \sin \beta_{1} \sin \beta_{2} \\
-\omega \mu_{0} \mu_{\mathrm{a}}-2 \omega \mu_{0} \mu_{\mathrm{b}} \cos \beta_{1} \cos \beta_{2} & -\omega \mu_{0} \mu_{\mathrm{b}} \sin \left(\beta_{1}+\beta_{2}\right) \\
0 & 0 \\
0 & 0
\end{array}
$$

0

and

$$
D_{\mathrm{EQ}}=1-\varkappa^{2} /\left(\omega^{2} \mu_{0} \varepsilon_{0} \mu_{\mathrm{a}} \varepsilon_{\mathrm{a}}\right) .
$$

Comparison of each element of $\mathbf{P}_{\mathrm{EQ}}$ with the corresponding element of the matrix $(1 / 2)\left(\mathbf{P}_{1}+\mathbf{P}_{2}\right)$ yields the following six equations:

(1) $\mu_{0}\left(\mu_{2 \|}-\mu_{2 \perp}\right) \sin \alpha \cos \alpha$

$$
=2 \omega \mu_{0} \mu_{\mathrm{b}} \sin \left(\beta_{1}+\beta_{2}\right),
$$




$$
\begin{aligned}
& \omega \mu_{0}\left(\mu_{1}+\mu_{2 \perp}\right)-\left(\varkappa^{2} / \omega \varepsilon_{0}\right)\left(1 / \varepsilon_{2}+1 / \varepsilon_{1 \perp}\right) \\
& +\omega \mu_{0}\left(\mu_{2 \|}-\mu_{2 \perp}\right) \sin ^{2} \alpha \\
& =2 \omega \mu_{0} \mu_{\mathrm{a}}-2 \varkappa^{2} / \omega \varepsilon_{0} \varepsilon_{\mathrm{a}}+4 \omega \mu_{0} \mu_{\mathrm{b}} \sin \beta_{1} \sin \beta_{2}, \\
& -\omega \mu_{0}\left(\mu_{2 \perp}+\mu_{1}\right)-\omega \mu_{0}\left(\mu_{2 \|}-\mu_{2 \perp}\right) \cos ^{2} \alpha \\
& =-2 \omega \mu_{0} \mu_{\mathrm{a}}-4 \omega \mu_{0} \mu_{\mathrm{b}} \cos \beta_{1} \cos \beta_{2}, \\
& \omega \varepsilon_{0}\left(\varepsilon_{1 \perp}-\varepsilon_{1 \|}\right) \sin \alpha \cos \alpha \\
& =-2 \omega \varepsilon_{0} \varepsilon_{\mathrm{b}} \sin \left(\alpha_{1}+\alpha_{2}\right), \\
& -\omega \varepsilon_{0}\left(\varepsilon_{2}+\varepsilon_{1 \perp}\right)+\left(\varkappa^{2} / \omega \mu_{0}\right)\left(1 / \mu_{1}+1 / \mu_{2 \perp}\right) \\
& +\omega \varepsilon_{0}\left(\varepsilon_{1 \perp}-\varepsilon_{1 \|}\right) \sin ^{2} \alpha \\
& =-2 \omega \varepsilon_{0} \varepsilon_{\mathrm{a}}+2 \varkappa^{2} / \omega \mu_{0} \mu_{\mathrm{a}}-4 \omega \varepsilon_{0} \varepsilon_{\mathrm{b}} \sin \alpha_{1} \sin \alpha_{2}, \\
& \omega \varepsilon_{0}\left(\varepsilon_{1 \perp}+\varepsilon_{2}\right)+\omega \varepsilon_{0}\left(\varepsilon_{1 \|}-\varepsilon_{1 \perp}\right) \cos ^{2} \alpha \\
& =2 \omega \varepsilon_{\mathrm{a}}+4 \omega \varepsilon_{0} \varepsilon_{\mathrm{b}} \cos \alpha_{1} \cos \alpha_{2} .
\end{aligned}
$$

Equations $(18 \mathrm{a}-\mathrm{f})$ hold for $\chi=0$ as well as for $\varkappa \neq 0$. By only considering the terms containing $\chi$ in $(18 b)$ and $(18 \mathrm{e})$, the equivalences

$$
\begin{aligned}
& \mu_{\mathrm{a}}=2\left(\mu_{1} \mu_{2 \perp}\right) /\left(\mu_{2 \perp}+\mu_{1}\right), \\
& \varepsilon_{\mathrm{a}}=2\left(\varepsilon_{2} \varepsilon_{1 \perp}\right) /\left(\varepsilon_{1 \perp}+\varepsilon_{2}\right)
\end{aligned}
$$

are obtained. Substituting $(19 a, b)$ in $(18 a-f)$ leads to the following prescription for the equivalent medium:

$\varepsilon_{\mathrm{b}}=(1 / 4)\left(\varepsilon_{1 \|}-\varepsilon_{1 \perp}\right), \quad \alpha_{1}+\alpha_{2}=2 \alpha$,

$\alpha_{1}-\alpha_{2}$

$$
=\arccos \left\{1-2\left(\varepsilon_{1 \perp}-\varepsilon_{2}\right)^{2} /\left[\left(\varepsilon_{1 \perp}+\varepsilon_{2}\right)\left(\varepsilon_{1 \perp}-\varepsilon_{1 \|}\right)\right]\right\},
$$

$\mu_{\mathrm{b}}=(1 / 4)\left(\mu_{2 \|}-\mu_{2 \perp}\right), \quad \beta_{1}+\beta_{2}=2 \alpha$,

$$
\beta_{1}-\beta_{2}
$$

$$
=\arccos \left\{1-2\left(\mu_{2 \perp}-\mu_{1}\right)^{2} /\left[\left(\mu_{2 \perp}+\mu_{1}\right)\left(\mu_{2 \perp}-\mu_{2 \|}\right)\right]\right\} .
$$

It is clear from (19e) and $(19 \mathrm{~h})$ that $\varepsilon_{\mathrm{EQ}}$ and $\boldsymbol{\mu}_{\mathrm{EQ}}$ are biaxial dyadics in general, since $\alpha_{1} \neq \alpha_{2}$ and $\beta_{1} \neq \beta_{2}$. In addition, (19e) and (19h) must be valid for real angles; therefore, $-1 \leqq \cos \left(\alpha_{1}-\alpha_{2}\right) \leqq 1$ and $-1 \leqq \cos \left(\beta_{1}-\beta_{2}\right)$ $\leqq 1$. Thus, the components of the permittivity and permeability dyadics must satisfy the conditions

$$
\begin{aligned}
& 0 \leqq\left(\varepsilon_{1 \perp}-\varepsilon_{2}\right)^{2} \leqq\left(\varepsilon_{1 \perp}+\varepsilon_{2}\right)\left(\varepsilon_{1 \perp}-\varepsilon_{1 \|}\right), \\
& 0 \leqq\left(\mu_{2 \perp}-\mu_{1}\right)^{2} \leqq\left(\mu_{2 \perp}+\mu_{1}\right)\left(\mu_{2 \perp}-\mu_{2 \|}\right),
\end{aligned}
$$

for the equivalences $(19 \mathrm{a}-\mathrm{h})$ to hold.

When $\varepsilon_{1 \perp} / \mu_{2 \perp}=\varepsilon_{1 \|} / \mu_{2 \|}=\varepsilon_{2} / \mu_{1}$, then $\alpha_{1}=\beta_{1}, \alpha_{2}=\beta_{2}$ and the biaxial dyadics $\boldsymbol{\varepsilon}_{\mathrm{EQ}}$ and $\boldsymbol{\mu}_{\mathrm{EQ}}$ have the same optic axes. If $\varepsilon_{1 \perp}=\varepsilon_{2}$, then $\alpha_{1}=\alpha_{2}$ and $\varepsilon_{\mathrm{EQ}}$ is a uniaxial dyadic with $\varepsilon_{\mathrm{a}}=\varepsilon_{2}$. Likewise, the condition $\mu_{2 \perp}=\mu_{1}$ yields $\beta_{1}=\beta_{2}$; hence $\boldsymbol{\mu}_{\mathrm{EQ}}$ is a uniaxial dyadic with $\mu_{\mathrm{a}}=\mu_{1}$ in that case.

With the specifications $(19 a-h)$, the effective medium properties of the laminated composite have been obtained, and electromagnetic fields inside this composite (within the long-wavelength approximation) can now be handled using vector-dyadic formalisms, e.g. $[2,5,6,8]$.

\section{Cross-Laminated Composites}

As an extension of the work presented here, a similar procedure is performed on a unit cell which has the permeability dyadic of one lamina orthogonal to the permittivity dyadic of the other; thus

$$
\begin{aligned}
& \boldsymbol{\varepsilon}_{1}=\varepsilon_{1 \perp} \mathbf{I}+\left(\varepsilon_{1 \|}-\varepsilon_{1 \perp}\right) \hat{\mathbf{c}}_{1} \hat{\mathbf{c}}_{1}, \quad \boldsymbol{\mu}_{1}=\mu_{1} \mathbf{I}, \\
& \boldsymbol{\varepsilon}_{2}=\varepsilon_{2} \mathbf{I}, \quad \boldsymbol{\mu}_{2}=\mu_{2 \perp} \mathbf{I}+\left(\mu_{2 \|}+\mu_{2 \perp}\right) \hat{\mathbf{c}}_{2} \hat{\mathbf{c}}_{2},
\end{aligned}
$$

where

$$
\begin{aligned}
& \hat{\mathbf{c}}_{1}=\hat{\boldsymbol{x}} \cos \alpha+\hat{\boldsymbol{y}} \sin \alpha, \\
& \hat{\mathbf{c}}_{2}=-\hat{\boldsymbol{x}} \sin \alpha+\hat{\boldsymbol{y}} \cos \alpha .
\end{aligned}
$$

By following the procedures outlined above, the equivalent medium properties can be obtained as

$\varepsilon_{\mathrm{a}}=2\left(\varepsilon_{2} \varepsilon_{1 \perp}\right) /\left(\varepsilon_{1 \perp}+\varepsilon_{2}\right)$,

$\mu_{\mathrm{a}}=2\left(\mu_{1} \mu_{2 \perp}\right) /\left(\mu_{2 \perp}+\mu_{1}\right)$,

$\varepsilon_{\mathrm{b}}=(1 / 4)\left(\varepsilon_{1 \|}-\varepsilon_{1 \perp}\right), \quad \alpha_{1}+\alpha_{2}=2 \alpha$,

$\alpha_{1}-\alpha_{2}=\arccos \left\{1-2\left(\varepsilon_{1 \perp}-\varepsilon_{2}\right)^{2} /\left[\left(\varepsilon_{1 \perp}+\varepsilon_{2}\right)\left(\varepsilon_{1 \perp}-\varepsilon_{1 \|}\right)\right]\right\}$,

$\mu_{\mathrm{b}}=-(1 / 4)\left(\mu_{2 \|}-\mu_{2 \perp}\right), \quad \beta_{1}+\beta_{2}=2 \alpha$,

$\beta_{1}-\beta_{2}=\pi-\arccos \left\{1-2\left(\mu_{1}-\mu_{2 \perp}\right)^{2} /\right.$

$$
\left./\left[\left(\mu_{2 \perp}+\mu_{1}\right)\left(\mu_{2 \perp}-\mu_{2 \|}\right)\right]\right\} \text {. }
$$

As in the previous case, $\boldsymbol{\varepsilon}_{\mathrm{EQ}}$ and $\boldsymbol{\mu}_{\mathrm{EQ}}$ are biaxial dyadics in general, since $\alpha_{1} \neq \alpha_{2}$ and $\beta_{1} \neq \beta_{2}$.

\section{Conclusion}

In summary, analysis was carried out for an alternating arrangement of laminae which have either an uniaxial permittivity dyadic or an uniaxial permeability dyadic. The unit cell is constituted by two laminae such that the ones with the uniaxial permittivity dyadic alternate with the ones with the uniaxial permeability dyadic through the stack. The 4-vector 
method was utilized to construct a characteristic matrix for the unit cell. After assuming that each lamina is electrically thin, the properties of an equivalent

[1] D. R. Lovett, Tensor Properties of Crystals, Adam Hilger, Bristol 1989.

[2] F. I. Fedorov, Theory of Gyrotropy (in Russian). Minsk: Nauka i Teknika 1976.

[3] A. Yariv and P. Yeh, Optical Waves in Crystals, Wiley, New York 1984

[4] A. Lakhtakia, V. K. Varadan, and V. V. Varadan, Effective Properties of a Periodic Chiral Arrangement of Idendical Biaxially Plates, J. Mater. Res. 4, 1511 (1989).

[5] A. Lakhtakia, V. V. Varadan, and V. K. Varadan, TimeHarmonic Electromagnetic Fields in Chiral Media, Springer-Verlag, Berlin 1989.

[6] H. C. Chen, Theory of Electromagnetic Waves. McGrawHill, New York 1983.

[7] Ph. Boulanger and M. Hayes, Electromagnetic Plane Waves in Anisotropic Media: An Approach using Bivectors, Philos. Trans. Royal Soc. London A 330, 335 (1990).

[8] J. van Bladel, Relativity and Engineering, Springer-Verlag, Berlin 1984. medium were obtained; both the permeability and the permittivity dyadics of the equivalent medium were shown to be biaxial.

[9] B. R. Chawla and H. Unz, Electromagnetic Waves in Moving Magnetoplasmas, University Press of Kansas, Lawrence 1971.

[10] V. H. Rumsey, Propagation in Generalized Gyrotropic Media, IEEE Trans. Antennas Propagat. 12, 83 (1964).

[11] P. S. Reese and A. Lakhtakia, A Periodic Chiral Arrangement of Thin Identical Bianisotropic Sheets: Effective Properties, Optik, vol. 86, pp. 47-50 (1990).

[12] H. Wöhler, G. Haas, M. Fritsch, and D. A. Mlynski, Faster $4 \times 4$ matrix Method for Uniaxial Inhomogeneous Media, J. Opt. Soc. Amer. A 5, 1554 (1988).

[13] J. L. Tsalamengas, Electromagnetic Fields of Elementary Dipole Antennas Embedded in Stratified General Gyrotropic Media, IEEE Trans. Antennas Propagat. 37, 399 (1989).

[14] H. Liew, An Alternative Derivation of the $4 \times 4$ Propagation Matrix Formalism, Optics Commun. 76, 302 (1990).

[15] H. Hochstadt, Differential Equations: A Modern Approach, Dover, New York 1975. 